\title{
Desigualdades na distribuição da cárie dentária no Brasil: uma abordagem bioética
}

\author{
Inequalities in the distribution of dental caries in Brazil: \\ a bioethical approach
}

Simone de M elo Costa ${ }^{1}$

$M$ auro $\mathrm{H}$ enrique $\mathrm{N}$ ogueira Guimarães deA breu $^{1}$

Mara Vasconcelos ${ }^{1}$

Rita de Cássia Gabrielli Souza Lima ${ }^{2}$

Marta Verdi 2

Efigênia Ferreira eFerreira ${ }^{1}$

${ }^{1}$ Departamento de

Odontologia, Universidade Estadual de M ontes Claros. Av. Dr. Ruy Bragas $/ \mathrm{n}$, Campus Universitário Professor Darcy Ribeiro, Vila M auricéia. 39401-089 M ontes Claros M G. smelocosta@gmail.com

${ }^{2}$ Departamento deSaúde Pública, Centro de Ciências daSaúde, U niversidade Federal de Santa Catarina.
Abstract The scope of this paper was to analyze the problem of dental caries as a public health issue of epidemiological relevance. It is a case study based on documentary analysis of national epidemiological surveyson oral health in theyears 1986, 2003 and 2010, with the Bioethics of Protection as an analytical support. The surveys revealed ine qualities between population groups; and such inequalities were analyzed with respect to the reduction of the disease in the younger population, access to oral health care, and tooth loss, which is the worst outcome for tooth decay. The good re sults presented in the surveys have not reached the whole population; data have shown inequalities regarding family income and Brazilian region. It was also found that from 2003 government interventions in protective actions such as the adoption of criteria of equity in the allocation of health resources and inclusion of vulnerable population have contributed to a reduction in caries. $\mathrm{N}$ evertheless, it is considered necessary update oral health care models by using Epidemiology as a structuring tool, and an interface with socially responsible protective bi oethics, since regional differences continued to be marked in the analysis.

Key words Epidemiology, Oral health, Public policies, Bioethics
Resumo Este artigo objetivou analisar a problemática da cárie dentária, enquanto uma questão desaúdepública derelevância epidemiológica, tendo como suporte analítico a visão da Bioética da Proteção. Trata-se de estudo de caso realizado a partir deanálise documental dosinquéritos epidemiológicos em saúde bucal, de basenacional, 1986, 2003 e2010. O slevantamentos apontam desigualdades entre grupos populacionais. Essa desigualdade foi analisada, com relação à redução da doença na população maisjovem, no acesso ao atendimento, ena perda dentária, pior desfecho para a cárie dentária. Os bons resultados apresentados, nos levantamentos, não atinge toda a população, apresentando desigualdades por renda familiar e região brasileira. Constatou-se a partir de 2003 intervenções do Estado em ações protetoras, como adoção de critérios de equidade na destinação dos recursosna saúdeeinclusão de populações em vulneração, contribuindo para redução da cárie. No entanto, considera-se necessário avançar mais nos model osassistenciaisem saúde bucal usando a Epidemiologia como uma ferramenta estruturante, já que as diferenças regionais ainda permaneceram marcantes, em interface com uma bioética protetora e socialmente responsável.

Palavras-chave Epidemiologia, Saúde bucal, Políticas públicas, Bioética 
Introdução

Ao longo do século XX observou-se que a doença não se limita a uma evidência apenas orgânica, natural e objetiva, mas engloba vivências individuais e coletivas de cada sociedade; portanto, é também realidade construída, sendo o doente um personagem social ${ }^{1}$. Os determinantes sociais de saúde são os fatores sociais, econômicos, culturais, étnicos/raciais, psicológicose comportamentais que influenciam no desencadeamento dos problemas de saúde e nos fatores de risco populacional².

A condição social tem sido, nos últimostempos, enfatizada como importante na avaliação da cárie dentária ${ }^{3-5}$. Sendo o indicador socioeconômico um dos fatores de risco para a cárie3. A baixa renda pode ser associada a menos acesso aos serviços odontológicos e aos produtos de higiene, também ao menor conhecimento sobre os corretos hábitos dehigiene bucal econsequentemente à alta prevalência e severidade de cárie dentária ${ }^{6}$. Estudos epidemiológicos constataram associação entre renda e pior condição de saúde bucal para a cárie $e^{7-11}$.

$\mathrm{Na}$ perspectiva da investigação cientifica a bioética aponta como uma das questões básicas, o valor social, ou seja, como a investigação pre tendemelhorar a saúde eo bem-estar dos indivíduos e das comunidades ${ }^{12}$. Se o beneficio não puder ser direto para os voluntários da pesquisa, pelo menos deverá ser para a categoria de portadores da condição a que ele pertence $\mathrm{e}^{13}$.

Com base no exposto, pretende-se nesse artigo, gerado no exercício de reflexão ética empre endido por profissionais de saúde, avaliar a existência e o grau de vulneração da população brasileira, frente à cárie dentária, doença de maior prevalência entre as que acometem a cavidade bucal. Para tanto, o arcabouço teórico da bioética da proteção foi utilizado como uma potente possibilidade de análise e reflexão.

\section{Procedimentos metodológicos}

Para empreender esta análise, utilizou-se um estudo de caso, de base documental numa perspectiva histórica. 0 estudo foi realizado por meio da avaliação de publicações, do Ministério da Saúde do Brasil, de dados e indicadores coletados nos Levantamentos Epidemiológicos de Saúde Bucal de base nacional de $1986^{14}, 2003^{15} \mathrm{e}$ $2010^{16}$. Os dados extraídos dos inquéritos epide miológicos para essa análise se relacionam à cá- rie dentária, medida pelo índiceCPOD, equere presenta a média do número total de dentes permanentes cariados (C) perdidos ( $P$ ) e obturados (0), em uma população ${ }^{14}$.

Buscou-se ancorar a discussão e análise dos dados no referencial da Bioética da Proteção. $\mathrm{Na}$ década de 1990, pesquisadores latino-americanos fundaram um campo da ética aplicada para a saúde pública, conhecido como bioética da proteção, com o propósito, entre outros, de enfrentar a questão da injustiça social e de seus efeitos na saúde de povos da América do Sul e Caribe, concomitante ao investimento de desconstrução de atitudes paternalistas estatais. A construção desta ferramenta analítica própria exigiu um esforço importante, pois, de um lado havia o compromisso de proteger atores sociais vulnerados e de acender o debate ético-político sobre a condição local de vulneração e de vulnerabilidade; de outro, havia o compromisso com a resistência incondicional ao paternalismo ${ }^{17}$.

A distinção do termo vulnerabilidade de vulneração équeo primeiro seria uma característica universal egenérica de qualquer ser humano, jáa vulneração se aplica aos casos específicos de se res humanos que independentemente de suas vontades não possuem meios ou capacidades para enfrentamento das contingências adversas ${ }^{18}$.

Esse campo da ética aplicada ao contexto latino-americano fundamenta-se no princípio da proteção que reconhece ser dever do Estado proteger os cidadãos contra ameaças, agressões externas, epidemiologias, calamidadesecatástrofes, partindo do pressuposto de que os cidadãos são susceptíveis e vulneráveis para se protegerem de tais eventos ${ }^{19}$ eosvulnerados, pessoas diretamente afe tadas em sua condição existencial de não poder exercer suas potencialidades (não empoderados) para ter uma vida digna e de qualidade ${ }^{20}$.

A bioética da proteção podeser definida como ética da responsabilidade social, agindo diante de situações de fragilização de ameaças às pessoas, legitimando o Estado como responsável em proteger a saúde das populações ${ }^{21}$ e trazendo re flexões queorientam a bioética para a coletividade em direção aos propósitos da saúde pública22, indicando as ferramentas para a reflexão da moralidade do agir humano ${ }^{23}$.

Nesse sentido, a proteção dos vulnerados relaciona-se com o princípio da equidade, ações sociais a favor dos mais necessitados diante da escassez de recursos para a saúde pública. A justificativa do princípio da proteção, que fundamenta a bioética da proteção, éoferecer proteção às pessoas quenão tenham capacidadede se pro- 
tegerem sozinhas, apesar de beneficiar qualquer pessoa, inclusiveaquela que não tem necessidade de ser protegida ${ }^{18}$.

O termo bioética da proteção traz o conceito da bioética (ética da vida) e o conceito de proteção ( prática consistente em amparar os necessitados, proteger os vulnerados) ${ }^{23}$. As ferramentas da bioética da proteção utilizam um método consistente em uma tripla função: a função descritiva (sentido e pertinência dos conceitos e das formas de argumentação), que descreve os conflitos da maneira mais racional e imparcial possível, podendo ser definida como função propriamente analítica; a função normativa (interrogação a morais e normas que controlam as interrelações), na medida em que se ocupa de enfrentar os conflitos, utilizando ferramentas consideradas mais adequadas para proscrever os comportamentos incorretos e prescrever os considerados corretos; e a função protetora, graças à correta articulação entre as funções descritiva e normativa e à sua aplicação a pessoas e contextos concretos ${ }^{22}$.

Trata-se, portanto, de um dispositivo de proteção moralmente legítimo construído na transformação e adaptação do corpo teórico prático da bioética tradicional21. A sua adaptação à contingência latino-americana e caribenhalhe conferelegitimidade, mas não existe a intenção de torná-la a "solução mágica" capaz de resolver todo e qualquer conflito ético entre os seres humanos ${ }^{23}$.

\section{A cárie dentária na população brasileira}

A partir de 1986, o M inistério da Saúde liderou e financiou inquéritos epidemiológicos em saúde bucal, em amostras da população brasileira, como ferramenta de planejamento eavaliação dos serviços nessa área. Na Tabela 1 são apresentados os levantamentos epidemiológicos de saúde bucal, cujos dados serão aqui analisados.

Publicações com dados desses levantamentos apontam desigualdades entre grupos populacionais ${ }^{14-16}$. Essa desigualdade será aqui analisada, com relação à redução da doença na população mais jovem, no acesso ao atendimento, e na perda dentária, pior desfecho para a cárie dentária. Pela ausência de dados disponíveis do levantamento de 2010, esse ano não será computado em algumas análises.

\section{A redução da doença na população e a desigualdade}

Um bom indicador para saúde bucal é o número de pessoas livres de cárie. Para essa avaliação a O rganização Mundial de Saúde (OM S) sugere os 12 anos, como idade índice ${ }^{24,25}$. O sresultados dos três inquéritos apontam, aos 12 anos, um aumento das crianças livres de cárie de 3,1\%, em 1986 para 31\% em 2003, al cançando 44\% em 2010, dado considerado favorável.

No entanto, numa análise pormenorizada, 0 bom resultado não atinge toda a população. Nos dados do levantamento de 1986, a distribuição de crianças livres de cárie por renda familiar mostrou diferenças entre crianças com renda de até dois salários mínimos (2,2\%) e as de 5 salários mínimos (6\%) sendo o percentual desse grupo quase três vezes maior que o primeiro. Considerando a região de moradia, as regiões Sul e Sudeste apresentaram o maior percentual de livres de cárie 4,8 e 5,3\%. No levantamento de $2003^{15}$, o mesmo fato é observado, quando 0 percentual variou de $24,1 \%$ ( N orte) a $37,5 \%$ (Sudeste), uma diferença de cerca de $60 \%$.

Outro dado que pode ser considerado nesta avaliação éa redução da cárie na população, que pode ser medida pela variação no CPOD. Na Tabela 2 observa-se o declínio continuado na prevalência da cárie dentária, nas faixas etárias que permitem essa comparação.

Tabela 1. Inquéritos epidemiológicos em Saúde Bucal, Brasil, 1986, 2003 e 2010.

\begin{tabular}{lll}
\hline Ano & \multicolumn{1}{c}{ Amostra } & Faixa etária \\
\hline 1986 & $\begin{array}{l}\text { 16 capitais brasileiras } \\
\text { representativas das macro }\end{array}$ & 6-12 anos \\
regiões - zona urbana & 35-19 anos \\
& & $50-59$ anos \\
& & $18-36$ meses \\
2003 & Municípios de diferentes & 5 anos \\
portes populacionais, zona & 12 anos \\
& urbana e rural & $15-19$ anos \\
& & $35-44$ anos \\
& & $65-74$ anos \\
2010 & Municípios de diferentes & 5 anos \\
portes populacionais, zona & 12 anos \\
urbana e rural & $15-19$ anos \\
& $35-44$ anos \\
& $65-74$ anos
\end{tabular}

Fonte: Brasil ${ }^{1416}$. 
Notadamente na idade de 12 anos, adolescentes (15-19 anos) e adultos (35-44 anos) o declínio é claramente observado. N esse intervalo de análise, não se pode deixar de referenciar o avanço da ciência odontológica, sobretudo com relação ao manejo da cárie dentária, as alterações no estabelecimento do diagnóstico e na de cisão de tratamento ${ }^{26}$. Mas, também merecem destaque nesse período, as políticas públicas significativas para a saúde bucal que foram estabe lecidas como a implantação do Sistema Ú nico de Saúde (SUS) 27, da Estratégia Saúde da Família (ESF) em 199428, das Equipes de Saúde Bucal (ESB) em $2000^{29}$ edas Diretrizes da Política Nacional de Saúde Bucal em $2004^{30}$.

Considerando o período 2002-2010, o investimento em saúde bucal foi expressivo, passando de 56 para 600 milhões de reais; de 4.261 para 20.300 Equipes de Saúde Bucal; de 41\% para 81\% de municípios com cirurgiões-dentistas em serviço; de 6 para 25 milhões de pessoas com cobertura ao tratamento especializado; mais cinco milhões de pessoas usufruindo da água fluore tada ${ }^{31}$. Estes resultados se associam a intervenções populacionais, aquelas capazes de modificar o perfil coletivo, com aumento no percentual de saudáveis, tais como fluoretação da água de consumo público, fluoretação de cremes dentais, aplicação tópica de flúor ${ }^{25}$.

De fato, a medida populacional que pode ser avaliada com estes dados é a adição de flúor na água de abastecimento público. No inquérito de $2003^{15}$, para crianças de 12 anos, o percentual de livres de cárie em cidades com água fluoretada foi de $37,5 \%$ enas demais, $23,64 \%$. Além da prevalência, a gravidade da doença também pode ser avaliada, por meio do percentual de dentes perdidos, que foi menor em cidades com água

Tabela 2. Índice CPO-D (cariados perdidos e obturados/dente) na população brasileira, segundo as faixas etárias examinadas, Brasil, 1986, 2003 e 2010.

\begin{tabular}{ccrrr}
\hline Índice & Faixa etária & 1986 & 2003 & 2010 \\
\hline & 12 anos & 6,9 & 2,78 & 2,1 \\
CPOD & $15-19$ anos & 12,7 & 6,17 & 4,2 \\
(dentes & $35-44$ anos & 22,5 & 20,13 & 16,3 \\
permanentes) & $50-59$ anos & 27,2 & $* *$ & $* *$ \\
& $65-74$ anos & $* *$ & 27,79 & 27,1
\end{tabular}

Fonte: BRASI L, $1988^{14} ; 2004^{15} ; 2010^{16}$. " D Dados não coletados. fluoretada $(4,41)$ do que em cidades sem água fluoretada $(7,99 \%)$.

No entanto, a análise feita por região brasileira, em todos os levantamentos analisados, mostrou variações na redução da cárie, com desvantagem para as regiões $\mathrm{N}$ orte, $\mathrm{N}$ ordeste e Centro-Oeste do Brasil, que apresentam maior carga da doença.

Como exemplo, na Tabela 3, observa-se um bom resultado com relação à cárie em crianças, incluindo o Brasil no grupo de países com baixa prevalência (CPOD entre 1,2 a 2,6). $M$ as, nas regiões N orte, N ordeste e Centro-O este foi observada média prevalência (CPOD entre2,7 a 4,4) ${ }^{16}$.

A diferença entre as regiões N orte e Sudeste, que em 1986 era de $20 \%$, passou a $26 \%$ em 2003, e $43 \%$ em 2010. Portanto, as diferenças regionais permaneceram em 2010. Além disso, o Nordeste tem menor proporção de dentes restaurados que - Sudeste, indicando além do maior ataque de cárieo menor acesso aos serviços da odontologia ${ }^{14,15}$.

0 acesso aos serviços de saúde a desigualdade

O IBGE em 1998 por meio da PNAD apontou problemas de acesso aos serviços de saúde no Brasil, sendo que 0 acesso a consultas odontológicas aumentou expressivamente no grupo com maior renda familiar. A pesar de 0 acesso ter sido maior em áreas urbanas, 32\% dessa população relatou nunca ter ido ao dentista. No geral, cerca de 5 milhões de pessoas referiram ter necessitado do serviço de saúde, mas não procuraram por falta de recursos financeiro ${ }^{32}$. De fato, o Levantamento de 1986 demonstrou queas pessoas com maior renda apresentaram menor necessidade de tratamento do que as de menor renda.

Tabela 3. Índice CPO-D (cariados perdidos e obturados/dente) e grau de prevalência na população brasileira, idade 12 anos, Brasil, 1986, 2003 e 2010.

\begin{tabular}{lcccc}
\hline \multicolumn{1}{c}{ Região } & 1986 & 2003 & 2010 & $\begin{array}{c}\text { Grau de } \\
\text { prevalência } \\
\text { (OMS) }\end{array}$ \\
\hline Norte & 7,4 & 3,1 & 3,2 & M édia \\
Nordeste & 6,9 & 3,2 & 3,1 & M édia \\
Sudeste & 5,9 & 2,3 & 1,7 & Baixa \\
Sul & 6,3 & 2,3 & 2,0 & Baixa \\
Centro-oeste & 8,5 & 3,2 & 3,1 & M édia \\
Brasil & 6,9 & 2,8 & 2,1 & Baixa
\end{tabular}

Fonte: BRASIL, $1988^{14} ; 2004^{15} ; 2010^{16}$. 
M elhorias nos indicadores de saúde bucal puderam ser vistosna pesquisa do IBGE em 2008 que apontou redução da população sem acesso ao dentista (de 18,7 a 11,6\%) num período de 10 anos. No entanto, a renda continuou a ser um diferencial já que, dos que declararam nunca ter ido ao dentista, 95,6\% pertenciam ao grupo de renda até 2 salários mínimos ${ }^{33}$.

Já no levantamento de $2003^{15}$, entre os adolescentes, nunca ter ido ao dentista foi um dado com maior frequência nas regiões Nordeste $(21,65 \%)$, N orte $(16,45 \%)$, seguindo-seas regiões Centro O este (12,01\%), Sudeste (10,13\%) e Sul $(5,34 \%)$. A procura pelo atendimento em caso de dor, na região Norte foi aproximadamente $56 \%$, enquanto que na região Sul foi $36 \%$.

Com relação à solução dos problemas apresentados, dados de $2003^{15}$ mostram percentuais menores de $50 \%$ nas regiões N orte e N ordeste e de $60 \%$ na região Centro-O este. As regiões Sul e Sudesteconseguiram solucionar cerca de $70 \%$ dos problemas apresentados.

Perda dentária como mutilação e desigualdade

O pior desfecho para a doença cárie é a perda dentária. Cientificamente, a extração dos dentesé considerada como uma decorrência do agravamento das doenças bucais mais prevalentes, entre elas a cárie. M as na prática, ocorre como solução definitiva para dor, sendo motivada principalmente pela falta de acesso ao serviço eà condição socioeconômica ${ }^{34}$. A incoerência reside no fato de que, a extração é realizada pelo serviço; portanto, para a extração não falta recurso nem acesso.

Considerando o sentido da palavra perda como "desaparecimento, extravio", entende-seque os dentes não desaparecem ou se extraviam, mas são efetivamente extraídos. Por outro lado, essa perda não seconfigura na maioria das vezes como tratamento indicado clinicamente, segundo os parâmetroscientíficosatuais. Sendo motivada por problemas sociais, a mutilação bucal pode ser vista também como uma mutilação social ${ }^{35}$.

Conforme o padrão da Organização Mundial de Saúde (OMS) e da Federação Dentária Internacional (FDI) ${ }^{36}$, para 0 ano de 2000, 85\% das pessoas com 18 anos deveriam estar com todos os dentes presentes na cavidade bucal. No Brasil, em 2003, o percentual para essa faixa etária foi $55,09 \%$. Além disso, este dado foi diferenciado entreas regiões. 0 percentual decompletamente dentados por macrorregiões, aos 18 anos foi de $39,1 \%$ (N orte), $45,1 \%$ (N ordeste), $66,5 \%$ (Sudeste e Sul) e 65,7\% (Centro-O este).

Na Tabela 4 observa-se que de 1986 a 2003, a redução da cárie dentária ocorreu de modo significativo somente para o grupo de adolescentes (CPOD 12,6 para 6,2). No grupo de adultos jovens a redução foi incipiente (CPOD 22,5 para 20,1). Já nos grupos de 50-59 anos e de 65-74 anos não foi possível avaliar os prováveis/improváveis benefícios por falta de dados.

No entanto, resultados preliminares, já disponibilizadospelo M inistério da Saúde, mostram melhoras em 201016. Para o grupo de adultos (35-44 anos), em um CPOD de 16,3, o componenteperdido foi de 7,3. M as entreidosos (65-74 anos), ainda não se observam essas melhoras. 0 CPOD foi 27,1 , praticamente o mesmo apresentado em $2003(27,8)$.

N esse panorama, vale a pena breve reflexão sobrea produção cultural de saúdebucal no Brasil, a partir do recurso da extração dentária. É fato que, historicamente, a extração é uma forte ancoragem de enfrentamento às realidades de dores dentárias produzidas nos mais diversos contextos sociais. É fato também que sua superação requer, entre outros, amplo serviço deaten-

Tabela 4. Índice de cárie (CPOD médio), componente perdido (P médio) percentual do perdido no CPOD, em adolescentes, adultos e idosos, Brasil, 1986, 2003.

\begin{tabular}{lcccccccc}
\hline \multirow{2}{*}{ Faixa etária } & \multicolumn{4}{c}{1986} & & \multicolumn{3}{c}{2003} \\
\cline { 2 - 4 } \cline { 7 - 9 } & CPOD & P & \% do CPOD & & CPOD & P & \% do CPOD \\
\hline $15-19$ anos & 12,6 & 1,9 & 15,2 & & 6,2 & 0,9 & 14,4 \\
$35-44$ anos & 22,5 & 14,9 & 66,5 & & 20,1 & 13,2 & 65,7 \\
$50-59$ anos & 27,2 & 23,4 & 85,9 & & $*$ & $*$ & $*$ \\
$65-74$ anos & $*$ & $*$ & $*$ & & 27,8 & 25,8 & 92,9
\end{tabular}

Fonte: BRASIL, 19884, 2004² . * Dados não coletados. 
ção secundária, que possa garantir a todos, por exemplo, a eliminação da dor diante deinfecções através de tratamento endodôntico. No entanto, considerando que o contexto brasileiro de marcantes desigual dades sociais se expressa no sujeito é importante refletir se o recurso de extração para a eliminação de sintomas é uma ação ética, no âmbito da proteção em saúde pública. Pois, examinando-o, em uma triangulação entre saúdebucal, sociedade/economia política ea experiência de dor (dor de dente como dor da vida), o que parece posto é que a extração é uma estraté gia produtora de uma nova condição de vulne ração: a denão ter dentes, como consequência de plano de tratamento. Ainda que sustentada por uma vertente positiva (eliminação da dor local) essa estratégia produz uma falsa expressão da economia política no sujeito e cria a ilusão de restabelecimento de saúde. Além disso, alimenta um farto mercado: o consumidor, já que a produção de saúde (diante de dentes perdidos) passa a gal gar expressão no consumo da técnica (próteses dentárias).

Saúde Bucal à luz da Bioética da Proteção

Sendo esta uma análise inicial, será possível utilizar como ferramenta da bioética de proteção ${ }^{22}$, a função descritiva, ou seja, procurar identificar e analisar criticamentea existência de conflitos, a partir do sentido e pertinência dos conceitos deste referencial.

o panorama de saúde bucal observado na análise dos dados permite identificar um conflito, relacionado à desigualdade: a desigualdade social de garantia de benefícios. Os benefícios observados com relação à prevalência da cárie, ao acesso e ao uso dos serviços odontológicos, bem como à gravidade do desfecho, representado pela mutilação dentaria, não são iguais para todos os brasileiros.

No que pese o investimento quetem sido feito em políticas publicas e financiamento, a de sigualdade permanece. Grupos vulnerados são identificados entre os de menor renda ou entre os que residem em regiões com menos desenvolvimento. Nesse sentido, os bons resultados observados com relação à cárie dentária, para parte da população significa que o Estado efetivamentetem protegido, por meio de políticas públicas, aqueles que se encontram acima de um limiar mínimo de condições de vida ${ }^{37}$. A hipóteseé que isso acontece porque as políticas públicas voltam-se para a perspectiva epidemiológica como a soberana enegam a perspectiva individual (com suas circunstancias singulares) e, sobretudo, a social.

Deste modo, faz-se necessário identificar os grupos populacionais que parecem estar historicamente condicionados ao estado de vulneração, aqueles que deverão ser protegidos até que saiam desta situação, respeitando a pluralidade cultural e moral. Não se trata aqui de estabelecer programas, muitas vezes criticados como paternalistas ou assistencialistas, que poderiam manter indivíduos ou populações sem responsabilidades. É de fundamental importância a discussão dos argumentos entre os capacitados e os vulnerados para que se estabeleçam contratos sociais entre eles ${ }^{23}$.

Um dos problemas observados foi a falta de acesso para determinados grupos. 0 levantamento de 1986, apesar de relevância para construção do perfil epidemiológico da população brasileira, não seconstituiu em elemento estratégico para a vigilância em saúde. A saúde bucal começou a se tornar preocupação do poder público a partir de 2000. Os resultados negativos da saúde bucal no Brasil incentivaram a inserção de ações da saúde bucal na Estratégia Saúde da Família, o que indicou que a direção da política de saúde bucal no Brasil estava começando a mudar ${ }^{29}$. 0 levantamento de saúde bucal, realizado em 2003, referenciou a implementação de novas políticas públicas, como a Política Nacional de Saúde Bucal (PNSB) ${ }^{30}$.

Contudo, a distribuição de recursos na saúde pública ainda leva à restrição do acesso aos serviços, o que dá origem a dilemas éticos em que a tomada de decisão deve considerar valores sociais, valores éticos ecritérios técnicos ${ }^{22}$. Osre cursos destinados à saúde pública no Brasil re presentam cerca de $3 \%$ do Produto Interno Bruto (PIB), com custo per capita de 120 a 150 dólares $^{18}$. Tais recursos, considerados baixos pela sociedade civil brasileira ${ }^{38}$, são um grande dificultador na aplicação dos princípios morais da igualdade de acesso, da integralidade da atenção e da universalidade do direito à saúde, previstos na Lei O rgânica da Saúde²7.

Com isto quer-se dizer que o Sistema Ú nico de Saúde (SUS) pouco avançou na efetivação de seus princípios doutrinários, o queconstitui grande problema bioético. Ainda perdura a "cultura dos limites", em que a seleção dos procedimentos se dá por questões orçamentárias, considerados escassos ${ }^{39}$. No entanto, o SUS apresenta al gumas características, queo torna protetor para vulnerados: a gratuidade, sob responsabilidade do estado; a vinculação, ou seja, a necessidade de 
proteção se tornando um dever do estado; a cobertura das necessidades, sobretudo dos vulnerados $^{21}$. A PN SB apontou a necessidade de organizar e planejar ações que pudessem facilitar 0 acesso dos cidadãos vulnerados à assistência odontológica, na tentativa de corrigir um dos aspectos da desigualdade observada ${ }^{30}$.

Nos últimos anos o governo tem tomado iniciativas de expandir o acesso aos serviços odontológicos não somente por meio da inclusão da saúde bucal na ESF, mas também mediante o Programa Brasil Sorridente, lançado em 2004, que incorpora assistência integral, expansão dos serviços e implantação de serviços especializados em outros níveis de atenção, ações de prevenção, promoção, e criação dos Centros de Especialidades Odontológicas (CEO) ${ }^{40}$.

A implantação de CEO se deu por meio de Portarias No o 599/GM de $2006^{41}$ que definiu crité rios, normas e requisitos para implantação e cre denciamento e a Portaria $\mathrm{N} \cong 600 / \mathrm{GM}-2006^{42}$ que instituiu o financiamento dos mesmos. Além de CEO, há também a implantação de Laboratórios Regionais de Prótese Dentária (LRPD) próprios do município ou terceirizados credenciadas para realização de próteses totais, próteses parciais removíveis e prótese coronária e intrarradiculares e as próteses fixas e as adesivas. Essa última medida foi tomada, diante do alto percentual (75\%) dos idosos desdentados e de uma parcela importante (30\%) dos adultos de 35-44 anos no levantamento epidemiológico nacional de2003.

Ainda com relação ao incentivo na confecção de próteses dentárias, o M inistério da Saúde em 2009 estabeleceu portarias $N .02 .374^{43}$ e $2.375^{44}$ GM/M S que alteraram o pagamento desse procedimento, aumentando o valor repassado ao município/Estado mensalmenteconformea produção. $\mathrm{O}$ estudo de Castro e $\mathrm{M}$ achado ${ }^{45}$ constatou quehouve criação de diversos incentivos, em especial, os programas prioritários na agenda Federal, como o Brasil Sorridente. Este aumentou os recursos para Saúde Bucal, de $R \$ 84,5$ milhões em 2003 para R \$553,2 milhões em 2006 (quase sete vezes) e ampliou o número de equipes de saú de bucal na ESF, de 6 mil em 2003 para 16 mil em $2006^{40}$.

No período decorrido de 2003 a 2008, o governo se empenhou em fortalecer as ações de monitoramento e avaliação na atenção primária à saúde (APS). Outro fato constatado foi que, no geral, em todas as regiões houve um crescimento a partir de 2003 nos valores per capita dos recursos federais para a APS. As regiões com os maiores valores per capita, Nordeste e N orte, apresentaram um aumento mais significativo a partir de $2003^{45}$.

No sentido deidentificar eproteger os vulnerados, esta parece ser uma decisão acertada, provendo financiamento diferenciado aos municípios mais pobres do país, localizados nessas macrorregiões, visando à equidade. No entanto, para a distribuição equânimedesaúdebucal (leiase: para resistir ao histórico "desaparecimento" dedentes em regiões vulneradas) , faz-senecessário o diálogo bioético no exercício do papel protetor do Estado.

Buscando ampliar a reflexão sobre essa necessária interlocução, Kottow ${ }^{46}$ sugeriu recentemente aliar uma vertente pragmática, ao campo da ética pública aplicada. $\mathrm{Na}$ sua perspectiva, as circunstâncias históricas parecem exigir uma bioética pública embasada sobre quatro pilares: ampla participação social; deliberação democrática; desenvolvimento da ética de proteção para empoderar a cidadania; e a proposta do pragmatismo estruturado, gerando instâncias e programas de enfrentamento das necessidades comunitárias e estímulo ao empoderamento dos excluídos ${ }^{46}$. N essa interlocução, a epidemiologia pode ser uma importante aliada, por ajudar na construção do quadro sanitário pertinente, por meio de atos legítimos e necessários para proteção dos necessitados ${ }^{21}$.

A partir do acesso aos serviços de saúde, no caso o odontológico, discute-sea necessidade de políticas públicas para corrigir situações de injustiça social, protegendo a saúde das pessoas principalmente daquelas ameaçadas em sua qualidade de vida ${ }^{47}$. N este sentido, a proteção pode ser entendida como cobertura das necessidades básicas ou essenciais ou indispensáveis moralmente legitimadas como a saúde, a educação, a alimentação, dentre outras necessidade ${ }^{21}$.

$M$ as, o acesso ao atendimento é necessário quando a população adoece e os dados mostraram que alguns grupos adoecem mais, os vulnerados. 0 acesso aos serviços de saúde precisa ser qualificado, modernizado cientificamenteea meta não pode ser outra senão "o melhor para todos", vulneráveis e vulnerados. A mutilação dentária é um fato quetem outras soluções. A perda dentaria pode ocorrer por indicação clinica, mas a mutilação dentaria faz parte da vida das pessoas, principalmente dos vulnerados. A desigualdade com relação à solução do problema existe e é perversa. A discussão ética entrevulnerados enão vulnerados é necessária para o estabelecimento de novo contrato. A condição subjetiva deve ser 
ultrapassada para que sejam estabelecidos que serviços devem ser oferecidos com recursos sanitários públicos e para quais pessoas ${ }^{37}$.

Estudo avaliativo contemporâneo demonstra que a cárie ainda tem sido considerada em nível mundial como uma das enfermidades de maior peso na história da morbidade bucal ${ }^{48}$. $\mathrm{Na}$ tentativa de compreender a vulneração no adoecer, o modelo ecológico de explicação do processo saúde e doença busca a articulação entre componentes causais das doenças, em relações de interação recíproca, respeitando os diferentes níveis. 0 modelo transpõe o nível individual de compreensão do desfecho, em direção ao nível populacional: o questionamento seria sempre voltado para a população e não para o indivíduo?

Embasados nessa teoria, Holst et al. ${ }^{6}$ argumentaram que as diferenças da experiência de cárie entrepopulações, de fato, têm si do mostradas pela associação entre cárie e escolaridade, nível socioeconômico, renda per capita, expectativa de vida, comportamentos relatados de saúde e estruturas de cuidados dos serviços de saúde bucal. Assim, as lesões cariosas se desenvolvem e progridem como resultado da estrutura social, contexto social, reações comportamentais e psicológicas, que aceleram o processo biológico.

Então não se pode falar de uma perspectiva epidemiológica desconectada da perspectiva social. 0 investimento público, apenas no atendimento, não conseguirá solucionar o problema da saúde bucal. O bservam-semelhoras, com certeza, capitaneadas por aqueles que têm melhores condições de vida. No caso em estudo, os bons resultados brasileiros estão sendo liderados pelas regiões Sul e Sudeste. 0 grupo dos vulnerados, já pouco beneficiado, não consegueusufruir completamente das medidas de proteção e terminam sendo mais prejudicados.

Com base na bioética da proteção, mecanismos de compensação pelo Estado devem ser ampliados, já que a saúde se caracteriza por ser um bem primário. Além do mais, seria o reconhecimento da desigualdade de vida, anterior à desigualdade do acesso, no atendimento de suas necessidades básicas. Em outra abordagem, a criação de mecanismos de compensação, por parte do Estado, seria o reconhecimento ético-político da condição dedesigual dadesocial vivenciada por grande parte da sociedade brasileira.

\section{Considerações finais}

A epidemiologia deve ter compromisso com a prática, o compromisso social. A pesquisa epidemiológica, como os inquéritos nacionais de saúde bucal, deve ser voltada para subsidiar e orientar as intervenções políticas na saúde, em ações de promoção, vigilância e avanços na integralidade da atenção à saúde. O levantamento epidemiológico do perfil da saúde bucal do brasileiro, especificamentea partir de 2003, decerto modo, contribuiu para a intervenção do Estado no sentido de implantar ações protetoras que poderiam reduzir o problema da cárie dentária. Foram constatadas mudanças com a introdução de critérios de equidade na destinação dos recursos da APS e busca de inclusão de populações em estado de vulneração e que eram negligenciadas pelas normas do sistema de saúde pública.

No entanto, a desigualdade, ainda permanece e parece ter se transformado num retrato banalizado, fruto do conformismo. o problema cárie dental ainda persiste no Brasil, exigindo a implantação de outras medidas pertinentes eque legitimam o papel do Estado como protetor da saúde pública, proporcionando aos cidadãos em estado de vulneração dispor de ações, além do investimento nos serviços odontológicos, conforme as suas necessidades.

Considera-se necessário avançar ainda mais nos modelos assistenciais em saúde bucal, mas para legitimar o papel protetor do Estado, esse deve ampliar as ações. 0 Estado devereconhecer e atuar nas desigualdades de vida entre as populações e desenvolver políticas públicas para a resolução do problema e o dado epidemiológico é uma boa ferramenta para uso nesse processo. 0 foco das ações deve priorizar os sujeitos vulnerados, com qualidade de vida insatisfatória.

Há necessidade de uma ética da responsabilidade pública, em que o Estado garanta aos cidadãos a liberdade para se constituírem como sujeitos ativos e participar das decisões políticas ${ }^{49}$. Isso porque os direitos foram e continuam sendo pensados, construídos e efetivados levando em consideração o processo de transformação da organização social ${ }^{50}$.

O exercício dereflexão bioética, aqui proposto, buscando demonstrar a importância da interface entre a epidemiologia e a ética aplicada, reafirmou a argumentação de Petrini et al. ${ }^{51}$ : a lentidão no efetivo compromisso público para com uma ética de responsabilidade, ao constituir-seem um conflito ético, reacende cotidianamente questões historicamente construídas que 
demandam enfrentamento público, a saber: a distribuição justa de riscos e benefícios entre a população; o acesso equitativo a cuidados desaúde e a redução das disparidades do estado de saúde.

\section{Referências}

1. M inayo MCS. Abordagem antropológica para avaliação de políticas sociais. Rev Saude Publica 1991; 25(3):233-238

2. Barata RB. Epidemiologia social. Rev bras epidemiol 2005; 8(1):7-17.

3. Baldani MH, Narvai PC, Antunes JLF. Cárie dentária e condições sócio-econômicas no Estado do Paraná, Brasil, 1996. Cad Saude Publica 2002; 18(3):755-763.

4. Comissão de Determinantes Sociais de Saúde (CDSS). Rumo a um modelo conceitual para análise e ação sobre os determinantes sociais de saúde. [serial on the internet]. $2005 \mathrm{M}$ aio [cited 2011 Oct 15]; Available from: http://www.determinantes. fiocruz.br

5. Cimões R, Caldas Júnior $A F$, Souza EHA, Gusmão ES. Influência da classe social nas razões clínicas das perdas dentárias. Cien Saude Colet 2007; 12(6):1691-1696.

6. Holst D, Schuller AA, Aleksejuniené J, Eriksen HM. Caries in population - a theoretical, causal approach. Eur J Oral Sci 2001; 109(3):143-148.

7. Brodeur JM, Payette M, Benigeri M, Gagnon PF, Olivier M, Chabot D. Dental caries in Quebec adults aged 35 to 44 years. J Can D ent Assoc 2000; 66(7):374379.

8. Doughan B, Kassak K, Bourgeois DM. Oral health status and treatment needs of 35-44-year old adults in Lebanon. Int Dent J 2000; 50(6):395-399.

9. Roberts-Thomson K, Stewart JF. Risk indicators of caries experience among young adults. Aust Dent J 2008; 53(2):122-127.

10. Skudutyte-Rysstad R, Sandvik L, Aleksejuniene J, Eriksen HM. Dental health and disease determinants among 35-year-olds in Oslo, Norway. Acta Odontol Scand 2009; 67(1):50-56.

11. Geyer S, Schneller T, M icheelis W. Social gradients and cumulative effects of income and education on dental health in the Fourth German Oral Health Study. Community Dent Oral Epidemiol 2010; 38(2): 120-128.

\section{Colaboradores}

SM Costa, MHGN de Abreu, M Vasconcellos, EF Ferreira participaram de todas as etapas: concepção, delineamento, análise einterpretação dos dados, redação do artigo. RCGS Lima, M Verdi contribuíram na revisão crítica do artigo. EF Ferreira orientou todas as fases do estudo.

12. Bedregal GP. Ética de la investigación en salud pública. ARS méd. 2006; 13(13):30-40.

13. Harris J. The principles of medical ethics and medical research. Cad Saude Publica 1999; 15(Supl. 1):S7S13.

14. Brasil. M inistério da Saúde (MS). Divisão Nacional de Saúde Bucal. Levantamento epidemiológico em saúde bucal: Brasil, zona urbana. Brasília: M S; 1988.

15. Brasil. M inistério da Saúde (M S). Secretaria de Atenção à Saúde. Departamento de Atenção Básica. Projeto SB Brasil 2003: condições de saúde bucal da população brasileira 2001-2003: resultados principais. Brasília: M S; 2004

16. Brasil. Ministério da Saúde (MS). Secretaria de Vigilância em Saúde. Secretaria de Assistência à Saúde. Departamento de Atenção Básica. Coordenação Nacional de Saúde Bucal. Pesquisa Nacional de Saúde Bucal - 2010. Nota para a imprensa. Brasília: M S; 2010.

17. Schramm FR. Bioética sem universalidade? Justificação de uma bioética latino-americana e caribenha de proteção. In: Garrafa V, Kottow M, Saada A. Bases Conceituais da Bioética: Enfoque Latino-Americano. São Paulo: Gaia; 2006. p. 143-157.

18. Boy R, Schramm FR. Bioética da proteção e tratamento de doenças genéticas raras no Brasil: o caso das doenças de depósito lisossomal. Cad Saude Publica 2009; 25(6):1276-1284.

19. Kottow M. The vulnerable and the susceptible. Bioethics 2003; 17(5):460-471.

20. Sen A. Desenvolvimento com liberdade. São Paulo: Companhia das Letras; 2000.

21. Schramm FR, Kottow M. Principios bioéticos en salud pública: limitaciones y propuestas. Cad Saude Publica 2001; 17(4):949-956.

22. Schramm FR. Bioética da proteção: justificativa e finalidades. Iatrós - Cadernos de Filosofia, Saúde e Cultura 2005; 1:121-130. 
23. Schramm FR. Bioética da Proteção: ferramenta valida para enfrentar problemas morais na era da globalização. Revista Bioética 2008; 16(1):11-13.

24. World Health Organization (WHO). Oral health surveys: basic methods. $4^{\text {th }}$ Edition. Geneva: ORH, EPID; 1997.

25. Petersen PE. The world oral health report, 2003. Ge neva: World Health Organization; 2003.

26. Elderton RJ. Changing the course of dental education to meet future requirements. J Can Dent Assoc 1997; 63(8):633-639.

27. Brasil. Conselho Nacional de Saúde. Lei 8.080 de 19 de setembro de 1990. Dispõe sobre as condições para a promoção, proteção e recuperação da saúde, a organização e o funcionamento dos serviços correspondentes e dá outras providências. Diário Oficial da União 1990; 20 set.

28. Brasil. Ministério da Saúde (MS). Saúde da Família: uma estratégia para a reorientação do modelo assistencial. Brasília: Ministério da Saúde; 1998.

29. Brasil. M inistério da Saúde. Programa Saúde da Família: equipes de saúde bucal. 2001. [acessado 2003 mar 25]. Disponível em: http://www.saude.gov.br

30. Brasil. M inistério da Saúde (MS). Secretaria de Atenção à Saúde. Departamento de Atenção Básica. Coordenação Nacional de Saúde Bucal. Diretrizes da Política Nacional de Saúde Bucal. Brasília: MS; 2004.

31. Brasil. M inistério da Saúde. SB BRASIL. 2010. [acessado 2010 jul 23]. Disponível em: http://dab.saude. gov.br/cnsb/

32. IBGE (Fundação Instituto Brasileiro de Geografia e Estatística). Pesquisa Nacional por Amostra de Domicílios - PNAD/BrasiL. 1998. Análise de Resultados. [acessado 2010 jul 23]. Disponível em: http://www. ibge.gov.br/home/estatistica/populacao/trabalhoe rendimento/pnad98/saude/analise.shtm

33. Fundação Instituto Brasileiro de Geografia e Estatística (IBGE). Pesquisa Nacional por Amostra de Domicílios - PNAD/Brasil. 2008. Um panorama da saúde no Brasil - Acesso e utilização dos serviços, condições de saúde e fatores de risco e proteção à saúde. [acessado 2010 jul 23].Disponível em: http://www. ibge.gov.br/home/estatistica/populacao/panorama saude_brasil_2003_2008/defaulttab_pdf_2.shtm

34. Vargas AMD, Paixão HH. Perda dentária e seu significado na qualidade de via de adultos usuários de serviço público de saúde bucal do Centro de Boa Vista, em Belo Horizonte. Cien Saude Colet 2005: 10(4):1015-1024.

35. Unfer B, Braun K, Silva CP, Pereira Filho LD. Autopercepção da perda de dentes em idosos. Interface Comun Saúde Educ 2006; 10(19):217-226.

36. Federation Dentaire Internacionale. Global goals for oral health in the year 2000. Int Dent J 1982; 32(1):74-77.

37. Ribeiro CDM, Rego S. Bioética clínica: contribuicões para a tomada de decisões em unidades de terapia intensiva neonatais. Cien Saude Colet 2008; 13(Supl. 2): 2239-2246.

38. Brasil. Ministério da Saúde. Conselho Nacional de Saúde (CNS). 0 desenvolvimento do Sistema Ú nico de Saúde: avanços, desafios e reafirmação dos seus princípios e diretrizes. Brasília: CNS; 2002.

39. Schramm FR. Bioética, economia e saúde: direito à assistência, justiça social, alocação de recursos. Rev Bras Cancerol 2000; 46:41-47.
40. Brasil. Ministério da Saúde (M S). Programa Brasil Sorridente. Política Nacional de Saúde Bucal. 2006. [acessado 2012 dez 2]. Disponível em: http://portal. saude.gov.br/portal/saude/visualizar_texto.cfm? idtxt $=21125$

41. Brasil. Portaria N N 599/GM de 23 de março de 2006 Define a implantação de Especialidades Odontológicas (CEO) e de Laboratórios Regionais de Próteses Dentárias (LRPDs) e estabelecer critérios, normas e requisitos para seu credenciamento. Ministério da Saúde. 2006. Diário Oficial da União 2006; mar 24.

42. Brasil. Portaria $\mathrm{N} 0600 / \mathrm{GM}$ de 23 de março de 2006. Institui o financiamento dos Centros de Especialidades Odontológicas. Diário Oficial da União 2006; mar 24.

43. Brasil. Portaria N .02 .374 GM /M S de 07 de outubro de 2009. Altera os valores dos procedimentos da Tabela de Procedimentos, M edicamentos, Órteses, Próteses e Materiais Especiais do Sistema Único de Saúde (SUS) realizados pelos Laboratórios Regionais de Próteses Dentárias (LRPD), segundo critérios estabelecidos pela Política Nacional de Saúde Bucal. Diário Oficial da União 2009; out 8.

44. Brasil. Portaria N .02 .375 GM /M S de 07 de outubro de 2009. Define os recursos anuais para o financiamento de procedimentos de prótese dentária. Diário Oficial da União 2009; out 8.

45. Castro ALB, Machado CV. A política de atenção primária à saúde no Brasil: notas sobre a regulaçãa e o financiamento federal. Cad Saude Publica 2010; 26(4):693-705.

46. Kottow M. Bioética pública: una propuesta. Revista bioética 2011; 19(1):61-76.

47. Pontes CAA, Schramm FR. Bioética da proteção e papel do Estado: problemas morais no acesso desigual à água potável. Cad Saude Publica 2004; 20(5): 1319-1327.

48. Rihs LB, Sousa MLR, Wada RS. Prevalência de cárie radicular em adultos e idosos na região sudeste do Estado de São Paulo, Brasil. Cad Saude Publica 2005; 21(1):311-316.

49. Gaudenzi P, Schramm FR. A transição paradigmática da saúde como um dever do cidadão: um olhar da bioética em Saúde Pública. Interface Comun Saúde Educ 2010; 14(33):243-255.

50. Lima RCGS, Severo DO, Verdi MIM, Da Ros MA. A construção do direito à saúde na Itália e no Brasil na perspectiva da bioética cotidiana. Saude soc. [online]. 2009 [cited 2011 Jun 07]; 18(1):118-130. Disponível em: http://www.scielo.br/scielo.php?script=sci arttext\&pid $=$ S0104- $12902009000100012 \&$ Ing=en\& $\bar{n} r \mathrm{~m}=\mathrm{iso}$

51. Petrini C, Gainotti S, Requena P. Personalism for public health ethics. Ann. Ist. Super. Sanità [online]. 2010[cited 2011 Jun 07]; 46(2):204-209. Available from: http://www.scielosp.org/scielo.php?script $=$ sci arttext\&pid $=$ S0021-25712010000200015\&lng= en\& $\overline{n r m}=i$ so

Artigo apresentado em 1305/2011

Aprovado em 03/09/2011

Versão final aprovada em 25/10/2011 